REVISTA DE LA

UNIÓN MATEMÁTICA ARGENTINA

Vol. 59, No. 1, 2018, Pages 193-204

Published online: November 22, 2017

\title{
ON PARTIAL ORDERS IN PROPER *-RINGS
}

\author{
JANKO MAROVT
}

\begin{abstract}
We study orders in proper $*$-rings that are derived from the corenilpotent decomposition. The notion of the C-N-star partial order and the S-star partial order is extended from $M_{n}(\mathbb{C})$, the set of all $n \times n$ complex matrices, to the set of all Drazin invertible elements in proper $*$-rings with identity. Properties of these orders are investigated and their characterizations are presented. For a proper $*$-ring $\mathcal{A}$ with identity, it is shown that on the set of all Drazin invertible elements $a \in \mathcal{A}$ where the core part of $a$ is an EP element, the C-N-star partial order implies the star partial order.
\end{abstract}

\section{INTRODUCTION}

Let $S$ be a semigroup. An involution ${ }^{*}$ on $S$ is called proper if $a^{*} a=a^{*} b=$ $b^{*} a=b^{*} b$, where $a, b \in S$, implies $a=b$. If a semigroup $S$ is equipped with a proper involution, then $S$ is called a proper *-semigroup. Natural special cases of proper $*$-semigroups are all proper $*$-rings (in particular, $M_{n}(\mathbb{C})$, the ring of all $n \times n$ complex matrices), with "properness" defined via $a a^{*}=0$ implying $a=0$. Drazin introduced in [2] a partial order, now known as the star partial order, on proper $*$-semigroups. The definition follows. Let $S$ be a proper $*$-semigroup. For $a, b \in S$, we write

$$
a \leq^{*} b \text { if } a^{*} a=a^{*} b \text { and } a a^{*}=b a^{*} .
$$

Recall that an element $a \in S$ is called regular when $a \in a S a$, and *-regular when there exists an element $a^{\dagger} \in S$ such that $a a^{\dagger} a=a, a^{\dagger} a a^{\dagger}=a^{\dagger},\left(a a^{\dagger}\right)^{*}=a a^{\dagger}$, and $\left(a^{\dagger} a\right)^{*}=a^{\dagger} a$. The element $a^{\dagger}$, which is unique if it exists, is known as the Moore-Penrose (generalized) inverse of $a$. We say that an element $a \in S$ has $a$ Drazin inverse $b \in S$ if

$$
a b=b a, \quad b=a b^{2}, \quad a^{k}=a^{k+1} b
$$

for some non-negative integer $k$ (see [1]). If $a$ has a Drazin inverse, then we say that $a$ is Drazin invertible and the smallest non-negative integer $k$ in $(2)$ is called the index $i(a)$ of $a$. It is well known that there is at most one $b$ such that 2 holds. The unique $b$, if it exists, will be denoted by $a^{D}$.

Let $\mathcal{A}$ be a ring with the (multiplicative) identity. We say that $a \in \mathcal{A}$ has the group inverse $a^{\#} \in \mathcal{A}$ if $x=a^{\#}$ satisfies the following equations: $a x a=a$,

2010 Mathematics Subject Classification. 06F25, 06A06, 15A09.

Key words and phrases. star partial order; Drazin inverse; core-nilpotent decomposition; ring. 
$x a x=x$, and $a x=x a$. Mitra introduced in [9] a partial order on the set of all $n \times n$ matrices over a field $\mathbb{F}$ which have the group inverse. This order, known as the sharp partial order, was generalized in [6] and independently in [13] to rings. The definition from [6] follows. Denote by $\mathcal{G}(\mathcal{A})$ the set of all elements in $\mathcal{A}$ which have the group inverse. For $a \in \mathcal{G}(\mathcal{A})$ and $b \in \mathcal{A}$, we write

$$
a \leq^{\sharp} b \text { if } a^{\#} a=a^{\#} b \text { and } a a^{\#}=b a^{\#} .
$$

In [6], the author proved that the sharp order $\leq \sharp$ is indeed a partial order on $\mathcal{G}(\mathcal{A})$.

Denote now by $\mathcal{N}(\mathcal{A})$ the set of all nilpotent elements in $\mathcal{A}$. Koliha gave in [4] an equivalent definition of the Drazin inverse for rings with identity. Namely, for $a, b \in \mathcal{A}, 2$ is equivalent to

$$
a b=b a, \quad b=a b^{2}, \quad a-a^{2} b \in \mathcal{N}(\mathcal{A}) .
$$

Moreover, the index $i(a)$ of $a$ is equal to the nilpotency index of $a-a^{2} b$. Note that group invertibility is a special case of Drazin invertibility (see [3]). Namely, if $i(a) \leq 1$, then the Drazin inverse of $a$ is exactly the group inverse of $a$.

Suppose $a \in \mathcal{A}$ has the Drazin inverse. It is known (see for example [15]) that then $a$ may be written as

$$
a=c+n
$$

where $c, n \in \mathcal{A}, c$ has the group inverse, $c n=n c=0$, and $n$ is nilpotent with index of nilpotency equal to $i(a)$. Then $c$ is called the core part of $a$ and $n$ the nilpotent part of $a$. Note that $c^{\#} n=0=n c^{\#}$ and therefore $c^{\#} a c^{\#}=c^{\#}, a c^{\#}=c^{\#} a$, and $a-a^{2} c^{\#}=n$. It follows (see (4) ) that $a^{D}=c^{\#}$. Since the Drazin inverse of every element in $\mathcal{A}$ is unique if it exists, we may conclude that $c$ and $n$ from (5) are unique. In fact,

$$
c=a^{2} a^{D} \quad \text { and } \quad n=a-a^{2} a^{D} .
$$

We refer to $c+n$ as the core-nilpotent decomposition of $a$.

It is known (see for example [10, Theorem 2.4.26]) that every matrix $A \in M_{n}(\mathbb{C})$ has the Drazin inverse. Thus, any matrix from $M_{n}(\mathbb{C})$ has the core-nilpotent decomposition (5). For a matrix $A \in M_{n}(\mathbb{C})$, let $\operatorname{Im} A$ denote the column space of $A$ and $\operatorname{Ker} A$ the null space of $A$. A matrix $A \in M_{n}(\mathbb{C})$ is said to be rangeHermitian (or EP) if $\operatorname{Im} A=\operatorname{Im} A^{*}$, or equivalently if $\operatorname{Ker} A=\operatorname{Ker} A^{*}$. Note that all Hermitian matrices and all non-singular matrices in $M_{n}(\mathbb{C})$ are range-Hermitian.

Let $\mathfrak{C}$ be the subset of all matrices in $M_{n}(\mathbb{C})$ whose core part is range-Hermitian. Let $A=C_{A}+N_{A}$ and $B=C_{B}+N_{B}$ be the core-nilpotent decompositions of $A$ and $B$, respectively, where $C_{A}$ is the core part of $A, C_{B}$ is the core part of $B, N_{A}$ is the nilpotent part of $A$, and $N_{B}$ is the nilpotent part of $B$. Mitra et al. introduced in [10] the following relations in $M_{n}(\mathbb{C})$.

Definition 1. Let $A, B \in M_{n}(\mathbb{C})$. We write $A \leq^{\sharp, *} B$ if $C_{A} \leq^{\sharp} C_{B}$ and $N_{A} \leq^{*} N_{B}$.

Definition 2. Let $A, B \in M_{n}(\mathbb{C})$. We write $A \leq{ }^{\circledast} B$ if $C_{A} \leq^{\sharp} C_{B}$ and $A \leq^{*} B$.

If $A \leq^{\sharp, *} B$, we say that $A$ is below $B$ with respect to the $C$ - $N$-star partial order, and if $A \leq{ }^{\circledast} B$, we say that $A$ is below $B$ with respect to the the $S$-star partial order. Mitra et al. noted in [10] that both $\leq^{\sharp, *}$ and $\leq^{\circledast}$ are partial orders, and 
that on $\mathfrak{C}$, the $\mathrm{C}-\mathrm{N}$-star partial order $\leq^{\sharp, *}$ implies the star partial order $\leq^{*}$, i.e. for $A, B \in \mathfrak{C}, A \leq^{\sharp * *} B$ yields $A \leq^{*} B$. They also posed the following open question.

Problem. What are necessary and sufficient conditions under which the S-star partial order $\leq^{\circledast}$ implies the C-N-star partial order $\leq^{\sharp, *}$ ?

The aim of this paper to generalize Definitions 1 and 2 to unital proper $*$-rings, study the properties of these orders, and solve Problem in a more general setting of proper *-rings with identity.

\section{Definitions ANd PRELIMINARY RESUlts}

Let $\mathcal{A}$ be a ring with identity. For a Drazin invertible $a \in \mathcal{A}$, we will denote by $c_{a}$ the core part of $a$ and by $n_{a}$ the nilpotent part of $a$. Mitra et al. extended in [10] the notion of the sharp order from the set $\mathcal{G}\left(M_{n}(\mathbb{F})\right)$ to the set $M_{n}(\mathbb{F})$ of all $n \times n$ matrices over a field $\mathbb{F}$. Namely, they introduced a relation on $M_{n}(\mathbb{F})$ using only the core part of matrices and ignoring the nilpotent part altogether. This relation has been recently generalized in [7] from $M_{n}(\mathbb{F})$ to the set of all Drazin invertible elements in rings with identity.

Definition 3. Let $a, b \in \mathcal{A}$ be Drazin invertible. The element $a$ is said to be below the element $b$ with respect to the the Drazin order if $c_{a} \leq^{\sharp} c_{b}$. When this happens, we write $a \leq^{D} b$.

The relation $\leq^{D}$ is a pre-order, i.e. it is reflexive and transitive, and it is not a partial order. Namely, the failure of anti-symmetry is due to the fact that the Drazin order ignores the nilpotent parts.

Unless stated otherwise, from now on let $\mathcal{A}$ be a proper $*$-ring with identity 1. Note that the C-N-star partial order, defined with Definition 1, is in fact a modification of the Drazin order on $M_{n}(\mathbb{C})$ so that the nilpotent parts are also involved. This modification transforms the Drazin pre-order to a partial order. Let us now generalize the notions of the C-N-star and the S-star partial orders from $M_{n}(\mathbb{C})$ to the set of Drazin invertible elements in a proper *-ring with identity.

Definition 4. Let $a, b \in \mathcal{A}$ be Drazin invertible. The element $a$ is said to be below the element $b$ with respect to the $C$ - $N$-star partial order if $c_{a} \leq^{\sharp} c_{b}$ and $n_{a} \leq^{*} n_{b}$. When this happens, we write $a \leq^{\sharp, *} b$.

Definition 5. Let $a, b \in \mathcal{A}$ be Drazin invertible. The element $a$ is said to be below the element $b$ with respect to the $S$-star partial order if $c_{a} \leq^{\sharp} c_{b}$ and $a \leq^{*} b$. When this happens, we write $a \leq{ }^{\circledast} b$.

Recall that the sharp order is a partial order on the set of all group invertible elements in a general ring with identity. Since the star order is also a partial order in a general proper $*$-ring, we obtain the following results.

Theorem 1. The order relation $\leq^{\sharp, *}$, defined with Definition 4, is a partial order on the set of all Drazin invertible elements in $\mathcal{A}$.

Theorem 2. The order relation $\leq{ }^{\circledast}$, defined with Definition 5 , is a partial order on the set of all Drazin invertible elements in $\mathcal{A}$. 
For an element $a$ in a $\operatorname{ring} \mathcal{A}$, we will denote by $a^{\circ}$ the right annihilator of $a$, i.e. the set $a^{\circ}=\{x \in \mathcal{A}: a x=0\}$. Similarly we denote the left annihilator ${ }^{\circ} a$ of $a$, i.e. the set ${ }^{\circ} a=\{x \in \mathcal{A}: x a=0\}$. Let us now present some auxiliary results.

Lemma 2.1. Let $\mathcal{A}$ be a proper $*$-ring and $a, b \in \mathcal{A}$. If $a \leq^{*} b$, then ${ }^{\circ} b \subseteq{ }^{\circ} a$ and $b^{\circ} \subseteq a^{\circ}$.

Proof. For $a, b \in \mathcal{A}$, let $a \leq^{*} b$. Let $z b=0, z \in \mathcal{A}$. From $a a^{*}=b a^{*}$, we have $0=z b a^{*}=z a a^{*}$. So, $z a a^{*} z^{*}=0$ and therefore $z a(z a)^{*}=0$. Since $\mathcal{A}$ is a proper *-ring, it follows that $z a=0$, i.e. ${ }^{\circ} b \subseteq{ }^{\circ} a$. The equation $a^{*} a=a^{*} b$ similarly implies $b^{\circ} \subseteq a^{\circ}$.

Lemma 2.2. Let $A \in M_{n}(\mathbb{C})$. Then $A$ is range-Hermitian (or EP) if and only if ${ }^{\circ} A={ }^{\circ}\left(A^{*}\right)$, which is equivalent to $A^{\circ}=\left(A^{*}\right)^{\circ}$.

Proof. Let $A, B \in M_{n}(\mathbb{C})$. By Lemma 2.1 in [8], we have ${ }^{\circ} A={ }^{\circ} B$ if and only if $\operatorname{Im} A=\operatorname{Im} B$, and $A^{\circ}=B^{\circ}$ if and only if $\operatorname{Ker} A=\operatorname{Ker} B$. It follows that ${ }^{\circ} A={ }^{\circ}\left(A^{*}\right)$ if and only if $\operatorname{Im} A=\operatorname{Im} A^{*}$ if and only if $\operatorname{Ker} A=\operatorname{Ker} A^{*}$ if and only if $A^{\circ}=\left(A^{*}\right)^{\circ}$.

We will use the following definition of EP elements in rings (see [5] ). An element $a$ of a ring $\mathcal{A}$ with involution ${ }^{*}$ is said to be EP if $a$ has the group inverse $a^{\#}$ and the Moore-Penrose inverse $a^{\dagger}$, and $a^{\#}=a^{\dagger}$.

Let $a \in \mathcal{A}$ be a $*$-regular element. Observe (see, e.g. [14]) that then ${ }^{\circ}\left(a^{*}\right)={ }^{\circ}\left(a^{\dagger}\right)$ and $\left(a^{*}\right)^{\circ}=\left(a^{\dagger}\right)^{\circ}$. For $b \in \mathcal{A}$, it follows that $a^{*} a=a^{*} b$ if and only if $a^{\dagger} a=a^{\dagger} b$, and similarly $a a^{*}=b a^{*}$ if and only if $a a^{\dagger}=b a^{\dagger}$. Since for an EP element $a, a^{\#}=a^{\dagger}$, we arrive at the following result.

Lemma 2.3. Let $a \in \mathcal{A}$ be an EP element. For $b \in \mathcal{A}$, we have $a \leq^{*} b$ if and only if $a \leq \sharp b$.

It turns out (see [11, 12]) that $a \in \mathcal{A}$ is an EP element if and only if $a \mathcal{A}=a^{*} \mathcal{A}$ and $a$ has the group inverse. For $a, b \in \mathcal{A}$, where $a$ and $b$ are regular, the following statement holds by [14, Lemmas 2.5 and 2.6]: ${ }^{\circ} a={ }^{\circ} b$ if and only if $a \mathcal{A}=b \mathcal{A}$. Note that for a group invertible $a \in \mathcal{A}, a^{*}$ has the group inverse $\left(a^{\#}\right)^{*}$. So, since a group invertible element is also regular, we may conclude that $a \in \mathcal{A}$ is $\mathrm{EP}$ if and only if $a$ has the group inverse and ${ }^{\circ} a={ }^{\circ}\left(a^{*}\right)$.

Let now $a \in \mathcal{A}$ be Drazin invertible. Since the core part $c_{a}$ of $a$ is group invertible and since all matrices in $M_{n}(\mathbb{C})$ are Drazin invertible, we may generalize the notion of the set $\mathfrak{C}$, of all matrices whose core part is range-Hermitian (or EP), from $M_{n}(\mathbb{C})$ to a $\operatorname{ring} \mathcal{A}$ with involution ${ }^{*}$ (see Lemma 2.2): Let $\mathfrak{C}^{\mathcal{A}}$ be the subset of all Drazin invertible elements $a \in \mathcal{A}$ where ${ }^{\circ} c_{a}={ }^{\circ}\left(c_{a}^{*}\right)$.

Mitra et al. observed that on $\mathfrak{C}=\mathfrak{C}^{M_{n}(\mathbb{C})}$, the $\mathrm{C}-\mathrm{N}$-star partial order $\leq^{\sharp \text {,* }}$ implies the star partial order $\leq^{*}$. In the next section, we will prove that a similar result holds also on $\mathfrak{C}^{\mathcal{A}}$ where $\mathcal{A}$ is a general proper $*$-ring with identity. First, let us present some useful tools.

The equality $1=e_{1}+e_{2}+\cdots+e_{n}$, where 1 is the identity of $\mathcal{A}, e_{1}, e_{2}, \ldots, e_{n}$ are idempotent elements in $\mathcal{A}$, and $e_{i} e_{j}=0$ for $i \neq j$, is called a decomposition of 
the identity of $\mathcal{A}$. Let $1=e_{1}+\cdots+e_{n}$ and $1=f_{1}+\cdots+f_{n}$ be two decompositions of the identity of $\mathcal{A}$. We have

$$
x=1 \cdot x \cdot 1=\left(e_{1}+e_{2}+\cdots+e_{n}\right) x\left(f_{1}+f_{2}+\cdots+f_{n}\right)=\sum_{i, j=1}^{n} e_{i} x f_{j} .
$$

Then any $x \in \mathcal{A}$ can be uniquely represented in the following matrix form:

$$
x=\left[\begin{array}{ccc}
x_{11} & \cdots & x_{1 n} \\
\vdots & \ddots & \vdots \\
x_{n 1} & \cdots & x_{n n}
\end{array}\right]_{e \times f},
$$

where $x_{i j}=e_{i} x f_{j} \in e_{i} \mathcal{A} f_{j}$. If $x=\left(x_{i j}\right)_{e \times f}$ and $y=\left(y_{i j}\right)_{e \times f}$, then $x+y=$ $\left(x_{i j}+y_{i j}\right)_{e \times f}$. Moreover, if $1=g_{1}+\cdots+g_{n}$ is a decomposition of the identity of $\mathcal{A}$ and $z=\left(z_{i j}\right)_{f \times g}$, then, by the orthogonality of the idempotents involved, $x z=\left(\sum_{k=1}^{n} x_{i k} z_{k j}\right)_{e \times g}$. Thus, if we have decompositions of the identity of $\mathcal{A}$, then the usual algebraic operations in $\mathcal{A}$ can be interpreted as simple operations between appropriate $n \times n$ matrices over $\mathcal{A}$. When $n=2$ and $p, q \in \mathcal{A}$ are idempotent elements, we may write

$$
x=p x q+p x(1-q)+(1-p) x q+(1-p) x(1-q)=\left[\begin{array}{ll}
x_{1,1} & x_{1,2} \\
x_{2,1} & x_{2,2}
\end{array}\right]_{p \times q} .
$$

Here $x_{1,1}=p x q, x_{1,2}=p x(1-q), x_{2,1}=(1-p) x q, x_{2,2}=(1-p) x(1-q)$.

If $\mathcal{A}$ is a ring with involution *, then we may by (7) write

$$
x^{*}=\left[\begin{array}{ccc}
x_{11}^{*} & \cdots & x_{n 1}^{*} \\
\vdots & \ddots & \vdots \\
x_{1 n}^{*} & \cdots & x_{n n}^{*}
\end{array}\right]_{f^{*} \times e^{*}}
$$

where this matrix representation of $x^{*}$ is given relative to the decompositions of the identity $1=f_{1}^{*}+\cdots+f_{n}^{*}$ and $1=e_{1}^{*}+\cdots+e_{n}^{*}$.

Let $a \in \mathcal{A}$ be Drazin invertible. It turns out (for details see [7]) that we may present the core nilpotent decomposition $c_{a}+n_{a}$ of $a$ in the following matrix form:

$$
a=\left[\begin{array}{cc}
c_{a} & 0 \\
0 & n_{a}
\end{array}\right]_{p \times p}
$$

where $p=a a^{D}$.

Remark. For a $\operatorname{ring} \mathcal{A}$ with involution ${ }^{*}, a \in \mathcal{A}$ is EP if and only if $a$ has the group inverse $a^{\#}$ and $a a^{\#}$ is self-adjoined (see [5, Theorem 7.3] or [11, Theorem 1.2]). Suppose ${ }^{\circ} c_{a}={ }^{\circ}\left(c_{a}^{*}\right)$. It follows that $c_{a}$ is then EP, which implies $\left(c_{a} c_{a}^{\#}\right)^{*}=c_{a} c_{a}^{\#}$. Recall (see the first section) that $a^{D}=c_{a}^{\#}$. Therefore,

$$
p=a a^{D}=\left(c_{a}+n_{a}\right) c_{a}^{\#}=c_{a} c_{a}^{\#},
$$

which implies that $p$ is a self-adjoined idempotent.

Let us conclude this section with a characterization [7, Theorem 1] of the Drazin order $\leq^{D}$ which we will use in the continuation. 
Proposition. Let $a, b \in \mathcal{A}$ be Drazin invertible. The following statements are then equivalent.

(i) $a \leq^{D} b$.

(ii) $a a^{D}=b a^{D}=a^{D} b=a^{D} a$.

(iii) There exists a decomposition of the identity $1=e_{1}+e_{2}+e_{3}$ such that

$$
a=\left[\begin{array}{ccc}
c_{1} & 0 & 0 \\
0 & c_{3} & c_{4} \\
0 & c_{5} & c_{6}
\end{array}\right]_{e \times e}, \quad b=\left[\begin{array}{ccc}
c_{1} & 0 & 0 \\
0 & c_{2} & 0 \\
0 & 0 & n_{2}
\end{array}\right]_{e \times e}
$$

where $n_{a}=c_{3}+c_{4}+c_{5}+c_{6}$ is the nilpotent part of $a, c_{2}$ has the group inverse, and $n_{2}$ is nilpotent.

\section{MAin RESUlts}

3.1. The $\mathbf{C}-\mathbf{N}$-star partial order. Recall that $\mathfrak{C}^{\mathcal{A}}$ is the set of all Drazin invertible elements $a \in \mathcal{A}$ where ${ }^{\circ} c_{a}={ }^{\circ}\left(c_{a}^{*}\right)$, i.e. the core part $c_{a}$ of $a$ is an EP element. We shall now present a new characterization of the $\mathrm{C}-\mathrm{N}$-star partial order on $\mathfrak{C}^{\mathcal{A}}$ where $\mathcal{A}$ is a proper $*$-ring with identity.

Theorem 3. Let $a, b \in \mathfrak{C}^{\mathcal{A}}$. Then $a \leq^{\sharp, *} b$ if and only if there exists a decomposition of the identity $1=e_{1}+e_{2}+e_{3}$, where $e_{1}, e_{2}, e_{3} \in \mathcal{A}$ are self-adjoined, such that

$$
a=\left[\begin{array}{ccc}
c_{1} & 0 & 0 \\
0 & 0 & 0 \\
0 & 0 & n_{1}
\end{array}\right]_{e \times e}, \quad b=\left[\begin{array}{ccc}
c_{1} & 0 & 0 \\
0 & c_{2} & 0 \\
0 & 0 & n_{2}
\end{array}\right]_{e \times e}
$$

where $c_{1}$ and $c_{2}$ have the group inverse, and $n_{1}$ and $n_{2}$ are nilpotent with $n_{1} \leq{ }^{*} n_{2}$.

Proof. Suppose $a, b \in \mathfrak{C}^{\mathcal{A}}$. Let $a=c_{a}+n_{a}$ and $b=c_{b}+n_{b}$ be the core-nilpotent decompositions of $a$ and $b$, respectively. By $(9)$ and Remark we may present element $a$ in the following matrix form:

$$
a=\left[\begin{array}{cc}
c_{a} & 0 \\
0 & n_{a}
\end{array}\right]_{p \times p}
$$

where $p=a a^{D}$ is self-adjoined.

Suppose $a \leq^{\sharp, *} b$. It follows that $a \leq^{D} b$ and therefore by Proposition there exists a decomposition of the identity $1=e_{1}+e_{2}+e_{3}$ such that

$$
a=\left[\begin{array}{ccc}
c_{1} & 0 & 0 \\
0 & c_{3} & c_{4} \\
0 & c_{5} & c_{6}
\end{array}\right]_{e \times e}, \quad b=\left[\begin{array}{ccc}
c_{1} & 0 & 0 \\
0 & c_{2} & 0 \\
0 & 0 & n_{2}
\end{array}\right]_{e \times e}
$$

where $n_{a}=c_{3}+c_{4}+c_{5}+c_{6}$ is the nilpotent part of $a, c_{2}$ has the group inverse, and $n_{2}$ is nilpotent. Since $c_{1}=a-n_{a}$, we may observe that $c_{1}=c_{a}$ is the core part of $a$. Note that for $d \in \mathcal{G}(\mathcal{A})$, we have ${ }^{\circ}\left(d^{\#}\right)={ }^{\circ} d$ and $\left(d^{\#}\right)^{\circ}=d^{\circ}$. It follows that $0=c_{2}^{\#} c_{a}=c_{a} c_{2}^{\#}=c_{2} c_{a}^{\#}=c_{a}^{\#} c_{2}$ and thus $\left(c_{a}+c_{2}\right)^{\#}=c_{a}^{\#}+c_{2}^{\#}$, i.e. $c_{a}+c_{2}$ is group invertible. So, since $\left(c_{a}+c_{2}\right) n_{2}=n_{2}\left(c_{a}+c_{2}\right)=0$, it follows that the core and the nilpotent parts of $b$ are $c_{a}+c_{2}=c_{b}$ and $n_{2}=n_{b}$, respectively. Observe that by the proof of Theorem 1 ((ii) implies (iii)) in [7] we may without loss of 
generality assume that $e_{1}=p=p^{*}, e_{2}=c_{2} c_{2}^{\#}$, and $e_{3}=1-e_{1}-e_{2}$. Since $b \in \mathfrak{C}^{\mathcal{A}}$, Remark implies $c_{b} c_{b}^{\#}$ is self-adjoined. So,

$$
\left(\left(c_{a}+c_{2}\right)\left(c_{a}+c_{2}\right)^{\#}\right)^{*}=\left(c_{a}+c_{2}\right)\left(c_{a}+c_{2}\right)^{\#}
$$

and therefore

$$
\left(c_{a} c_{a}^{\#}\right)^{*}+\left(c_{2} c_{2}^{\#}\right)^{*}=c_{a} c_{a}^{\#}+c_{2} c_{2}^{\#} .
$$

Recall that $a \in \mathfrak{C}^{\mathcal{A}}$. So, $\left(c_{a} c_{a}^{\#}\right)^{*}=c_{a} c_{a}^{\#}$ which yields that $\left(c_{2} c_{2}^{\#}\right)^{*}=c_{2} c_{2}^{\#}$. We may conclude that the idempotents $e_{1}, e_{2}$, and $e_{3}$ are all self-adjoined.

Let us now show that in (11), $c_{3}=c_{4}=c_{5}=0$. From $a \leq^{\sharp, *} b$, we have $n_{a} \leq^{*} n_{b}$. Since $n_{a}=c_{3}+c_{4}+c_{5}+c_{6}$ and $n_{b}=n_{2}$, it follows by Lemma 2.1 that

$$
{ }^{\circ} n_{2} \subseteq{ }^{\circ}\left(c_{3}+c_{4}+c_{5}+c_{6}\right) \text { and } n_{2}^{\circ} \subseteq\left(c_{3}+c_{4}+c_{5}+c_{6}\right)^{\circ} \text {. }
$$

We have $c_{3}+c_{4}+c_{5}+c_{6}=e_{2} a e_{2}+e_{2} a e_{3}+e_{3} a e_{2}+e_{3} a e_{3}$ and $n_{2}=e_{3} b e_{3}$. Since $e_{2} e_{3}=0=e_{3} e_{2}$, we obtain $e_{2} n_{2}=0=n_{2} e_{2}$, i.e. $e_{2} \in{ }^{\circ} n_{2} \cap n_{2}^{\circ}$. So, by (12),

$$
e_{2} \in{ }^{\circ}\left(c_{3}+c_{4}+c_{5}+c_{6}\right) \cap\left(c_{3}+c_{4}+c_{5}+c_{6}\right)^{\circ}
$$

and therefore

$$
0=e_{2}\left(c_{3}+c_{4}+c_{5}+c_{6}\right)=e_{2} a e_{2}+e_{2} a e_{3}=c_{3}+c_{4}
$$

and

$$
0=\left(c_{3}+c_{4}+c_{5}+c_{6}\right) e_{2}=e_{2} a e_{2}+e_{3} a e_{2}=c_{3}+c_{5} .
$$

So, $c_{4}=c_{5}=-c_{3}$. Note that $c_{4} \in e_{2} \mathcal{A} e_{3}$ and $c_{5} \in e_{3} \mathcal{A} e_{2}$. Since $e_{2} \mathcal{A} e_{3} \cap e_{3} \mathcal{A} e_{2}=$ $\{0\}$, we may conclude that $0=c_{3}=c_{4}=c_{5}$. If we denote $c_{6}=n_{1}$, we obtain the matrix form (10) of $a$ and $b$.

Conversely, let $a, b \in \mathfrak{C}^{\mathcal{A}}$ be of the matrix form $(10)$. Since $c_{1}$ is group invertible and $n_{1}$ is nilpotent with $c_{1} n_{1}=n_{1} c_{1}=0$, the uniqueness of the core-nilpotent decomposition implies $c_{a}=c_{1}$ and $n_{a}=n_{1}$. Similarly, $c_{b}=c_{1}+c_{2}$ and $n_{b}=n_{2}$. By Proposition it follows that $a \leq^{D} b$, i.e. $c_{a} \leq^{\sharp} c_{b}$. Therefore, since by assumption $n_{a} \leq^{*} n_{b}$, we may conclude that $a \leq^{\sharp, *} b$.

The nilpotent part of a Drazin invertible element $a \in \mathcal{A}$ is by (6), $n_{a}=a-$ $a^{2} a^{D}=a-a a^{D} a$. Thus, directly by Definitions 3 and 4 , we obtain another characterization of the $\mathrm{C}-\mathrm{N}$-star partial order on a proper $*$-ring $\mathcal{A}$ with identity.

Theorem 4. Let $a, b \in \mathcal{A}$ be Drazin invertible. Then $a \leq^{\sharp, *} b$ if and only if $a \leq^{D} b$ and $a-a a^{D} a \leq^{*} b-b b^{D} b$.

With the next result we will show that on $\mathfrak{C}^{\mathcal{A}}$, the $\mathrm{C}$-N-star partial order $\leq_{\sharp}^{\sharp, *}$ implies the star partial order $\leq *$.

Theorem 5. Let $a, b \in \mathfrak{C}^{\mathcal{A}}$. If $a \leq^{\sharp, *} b$, then $a \leq^{*} b$.

Proof. Suppose $a, b \in \mathfrak{C}^{\mathcal{A}}$ and $a \leq^{\sharp, *} b$, i.e. $c_{a} \leq^{\sharp} c_{b}$ and $n_{a} \leq^{*} n_{b}$. The star partial order (1) and the sharp partial order (3) are by Lemma 2.3 equivalent on the set of EP elements in $\mathcal{A}$. Since the core part $c_{a}$ of $a$ is an EP element, we may conclude that $c_{a} \leq^{*} c_{b}$. It follows that $c_{a}^{*} c_{a}=c_{a}^{*} c_{b}$ and $c_{a} c_{a}^{*}=c_{b} c_{a}^{*}$. Also, $n_{a}^{*} n_{a}=n_{a}^{*} n_{b}$ and $n_{a} n_{a}^{*}=n_{b} n_{a}^{*}$. Since $a=c_{a}+n_{a}$ and $b=c_{b}+n_{b}$, we obtain

$$
a^{*} a=c_{a}^{*} c_{a}+c_{a}^{*} n_{a}+n_{a}^{*} c_{a}+n_{a}^{*} n_{a} \quad \text { and } \quad a^{*} b=c_{a}^{*} c_{b}+c_{a}^{*} n_{b}+n_{a}^{*} c_{b}+n_{a}^{*} n_{b} .
$$


Observe that for any $d \in \mathcal{A},{ }^{\circ} d={ }^{\circ}\left(d^{*}\right)$ if and only if $d^{\circ}=\left(d^{*}\right)^{\circ}$. So, since $a \in \mathfrak{C}^{\mathcal{A}}$ and therefore ${ }^{\circ} c_{a}={ }^{\circ}\left(c_{a}^{*}\right)$, we have $c_{a}^{*} n_{a}=0=n_{a}^{*} c_{a}$. By Theorem 3, elements $a$ and $b$ have the matrix representation (10), where $c_{1}=c_{a}$ is the core part of $a$, $n_{1}=n_{a}$ is the nilpotent part of $a, c_{1}+c_{2}=c_{b}$ is the core part of $b$, and $n_{2}=n_{b}$ is the nilpotent part of $b$. Clearly, by 10 we have $c_{1} n_{2}=0$, i.e. $c_{a} n_{b}=0$, which yields $0=c_{a}^{*} n_{b}$. Similarly, $\left(c_{1}+c_{2}\right) n_{1}=0$, i.e. $c_{b} n_{a}=0$. Since $b \in \mathfrak{C}^{\mathcal{A}}$ and therefore ${ }^{\circ} c_{b}={ }^{\circ}\left(c_{b}^{*}\right)$, we obtain $0=c_{b}^{*} n_{a}$ and thus $0=n_{a}^{*} c_{b}$. By 13 , we may conclude that

$$
a^{*} a=a^{*} b
$$

We may similarly prove that $a a^{*}=b a^{*}$. Therefore, $a \leq^{*} b$.

3.2. The S-star partial order. With Theorem 5 we showed that on $\mathfrak{C}^{\mathcal{A}}$, where $\mathcal{A}$ is a proper $*$-ring with identity, the $\mathrm{C}$-N-star partial order $\leq \sharp, *$ implies the star partial order $\leq^{*}$. It follows (compare Definitions 4 and 5 that the $\mathrm{C}-\mathrm{N}$-star partial order implies also the S-star partial order $\leq{ }^{\circledast}$. With the next theorem, we will present some new characterizations of the $\mathrm{C}-\mathrm{N}$-star partial order and thus find some conditions under which the S-star partial order implies the C-N-star partial order.

Theorem 6. Let $a, b \in \mathfrak{C}^{\mathcal{A}}$, let $k=\max \{i(a), i(b)\}$, and suppose $a \leq^{\circledast} b$. The following statements are then equivalent.

(i) $a \leq \sharp, * b$

(ii) $b^{k} a b b^{D}=b^{k} a, b b^{D} a b^{k}=a b^{k}$, and $b b^{D} a=a a^{D} a$

(iii) ${ }^{\circ}\left(b^{k}\right) \subseteq{ }^{\circ}\left(a b^{k}\right)$ and $b^{k} a=a^{k+1}$

(iv) ${ }^{\circ}\left(\left(b^{k}\right)^{*}\right) \subseteq{ }^{\circ}\left(\left(b^{k} a\right)^{*}\right)$ and $a b^{k}=a^{k+1}$

(v) $a b^{k}=b^{k} a=a^{k+1}$

Proof. Some steps of the proof will be similar to the corresponding steps in the proof of Theorem 8 in [7. For the sake of completeness, we will not skip these details. Let $a \leq^{\sharp, *} b$. By Theorem 3 , there exists a decomposition of the identity $1=e_{1}+e_{2}+e_{3}$, where $e_{1}, e_{2}, e_{3} \in \mathcal{A}$ are self-adjoined, such that

$$
a=\left[\begin{array}{ccc}
c_{1} & 0 & 0 \\
0 & 0 & 0 \\
0 & 0 & n_{1}
\end{array}\right]_{e \times e}, \quad b=\left[\begin{array}{ccc}
c_{1} & 0 & 0 \\
0 & c_{2} & 0 \\
0 & 0 & n_{2}
\end{array}\right]_{e \times e}
$$

where $c_{1}$ and $c_{2}$ have the group inverse, and $n_{1}$ and $n_{2}$ are nilpotent with $n_{1} \leq{ }^{*} n_{2}$. Note that $c_{a}=c_{1}, n_{a}=n_{1}, c_{b}=c_{1}+c_{2}$, and $n_{b}=n_{2}$. Since $k=\max \{i(a), i(b)\}$, we have $n_{1}^{k}=0=n_{2}^{k}$ and therefore

$$
a^{k+1}=\left[\begin{array}{ccc}
c_{1}^{k+1} & 0 & 0 \\
0 & 0 & 0 \\
0 & 0 & 0
\end{array}\right]_{e \times e} \text { and } \quad b^{k}=\left[\begin{array}{ccc}
c_{1}^{k} & 0 & 0 \\
0 & c_{2}^{k} & 0 \\
0 & 0 & 0
\end{array}\right]_{e \times e}
$$


(i) $\Rightarrow\left(\right.$ ii): Observe that $a^{D}=c_{1}^{\#}=\left[\begin{array}{ccc}c_{1}^{\#} & 0 & 0 \\ 0 & 0 & 0 \\ 0 & 0 & 0\end{array}\right]_{e \times e}$ and $b^{D}=\left(c_{1}+c_{2}\right)^{\#}=c_{1}^{\#}+c_{2}^{\#}=$ $\left[\begin{array}{ccc}c_{1}^{\#} & 0 & 0 \\ 0 & c_{2}^{\#} & 0 \\ 0 & 0 & 0\end{array}\right]_{e \times e}$. We obtain

$$
b^{k} a b b^{D}=\left[\begin{array}{ccc}
c_{1}^{k} c_{1} c_{1} c_{1}^{\#} & 0 & 0 \\
0 & 0 & 0 \\
0 & 0 & 0
\end{array}\right]_{e \times e}=\left[\begin{array}{ccc}
c_{1}^{k+1} & 0 & 0 \\
0 & 0 & 0 \\
0 & 0 & 0
\end{array}\right]_{e \times e}=b^{k} a
$$

and similarly $b b^{D} a b^{k}=c_{1}^{k+1}=a b^{k}$, and $b b^{D} a=c_{1}=a a^{D} a$.

(i) $\Rightarrow$ (iii): Clearly, by (14) and (15), $b^{k} a=c_{1}^{k+1}=a^{k+1}$. Let $z \in{ }^{\circ}\left(b^{k}\right)$, i.e. $z b^{k}=0$. Since $b^{k}=c_{1}^{k}+c_{2}^{k}$, we obtain $z c_{1}^{k}+z c_{2}^{k}=0$ and thus, $z c_{1}^{k+1}+z c_{2}^{k} c_{1}=0$. Note that $c_{1} c_{2}=c_{2} c_{1}=0$. So, $z c_{1}^{k+1}=0$. Observe that $a b^{k}=c_{1}^{k+1}$. Therefore, $z \in{ }^{\circ}\left(a b^{k}\right)$, i.e. ${ }^{\circ}\left(b^{k}\right) \subseteq{ }^{\circ}\left(a b^{k}\right)$.

(i) $\Rightarrow$ (iv): Again, clearly, $a b^{k}=a^{k+1}=c_{1}^{k+1}=b^{k} a$. Let $z \in{ }^{\circ}\left(\left(b^{k}\right)^{*}\right)$. Thus $z\left(c_{1}^{k}\right)^{*}+z\left(c_{2}^{k}\right)^{*}=0$ and therefore $z\left(c_{1}^{k+1}\right)^{*}+z\left(c_{2}^{k}\right)^{*} c_{1}^{*}=0$. Since $c_{1} c_{2}=0$, we have $c_{2}^{*} c_{1}^{*}=0$ and hence $z \in{ }^{\circ}\left(\left(c_{1}^{k+1}\right)^{*}\right)={ }^{\circ}\left(\left(b^{k} a\right)^{*}\right)$.

(i) $\Rightarrow(\mathrm{v})$ : It follows directly by (14) and $(15)$.

(ii) $\Rightarrow$ (i): Let $a \leq^{\circledast} b$, i.e. $c_{a} \leq^{\sharp} c_{b}$ and $a \leq^{*} b$, and let $b^{k} a b b^{D}=b^{k} a, b b^{D} a b^{k}=$ $a b^{k}$, and $b b^{D} a=a a^{D} a$. We will show that then $n_{a} \leq^{*} n_{b}$. By (9),

$$
b=\left[\begin{array}{cc}
c_{b} & 0 \\
0 & n_{b}
\end{array}\right]_{q \times q}
$$

where $q=b b^{D}=c_{b} c_{b}^{\#}$. Note (see Remark) that $q=q^{*}$. Also, since $n_{b}$ is the nilpotent part of $b$, we have $n_{b}^{k}=0$ and therefore

$$
b^{k}=\left[\begin{array}{cc}
c_{b}^{k} & 0 \\
0 & 0
\end{array}\right]_{q \times q} .
$$

Let $a=\left[\begin{array}{ll}a_{1} & a_{2} \\ a_{3} & a_{4}\end{array}\right]_{q \times q}$. From $b^{k} a b b^{D}=b^{k} a$, we obtain $0=b^{k} a\left(1-b b^{D}\right)=$ $b^{k} a(1-q)$ and thus

$$
0=\left[\begin{array}{cc}
c_{b}^{k} & 0 \\
0 & 0
\end{array}\right]_{q \times q}\left[\begin{array}{ll}
a_{1} & a_{2} \\
a_{3} & a_{4}
\end{array}\right]_{q \times q}\left[\begin{array}{cc}
0 & 0 \\
0 & 1-q
\end{array}\right]_{q \times q}=\left[\begin{array}{cc}
0 & c_{b}^{k} a_{2}(1-q) \\
0 & 0
\end{array}\right]_{q \times q} .
$$

So, $c_{b}^{k} a_{2}(1-q)=0$. This yields $c_{b}^{k} a_{2}=0$ since $a_{2} \in q \mathcal{A}(1-q)$. It follows that

$$
0=\left(c_{b}^{\#}\right)^{k} c_{b}^{k} a_{2}=q^{k} a_{2}=a_{2} .
$$

Similarly, from $b b^{D} a b^{k}=a b^{k}$ we obtain $0=(1-q) a b^{k}$ and therefore $0=(1-q) a_{3} q^{k}$. So, $a_{3}=0$ since $a_{3} \in(1-q) \mathcal{A} q$. Thus,

$$
a=\left[\begin{array}{cc}
a_{1} & 0 \\
0 & a_{4}
\end{array}\right]_{q \times q} .
$$

Since $a_{1} \in q \mathcal{A} q$ and $a_{4} \in(1-q) \mathcal{A}(1-q)$, we have

$$
a_{4}=(1-q) a_{1}+(1-q) a_{4}=(1-q)\left(a_{1}+a_{4}\right)=(1-q) a=a-b b^{D} a .
$$


The assumption $b b^{D} a=a a^{D} a$ yields $a_{4}=a-a^{2} a^{D}$, which is by (6) exactly the nilpotent part of $a$. So, $a_{1}=a-a_{4}=a^{2} a^{D}$ is the core part of $a$ and thus $a$ may be presented in the following matrix form:

$$
a=\left[\begin{array}{cc}
c_{a} & 0 \\
0 & n_{a}
\end{array}\right]_{q \times q} .
$$

Recall that $a \leq^{*} b$, i.e. $a^{*} a=a^{*} b$ and $a a^{*}=b a^{*}$. Since $q=q^{*}$, the first equation yields

$$
\left[\begin{array}{cc}
c_{a}^{*} & 0 \\
0 & n_{a}^{*}
\end{array}\right]_{q \times q}\left[\begin{array}{cc}
c_{a} & 0 \\
0 & n_{a}
\end{array}\right]_{q \times q}=\left[\begin{array}{cc}
c_{a}^{*} & 0 \\
0 & n_{a}^{*}
\end{array}\right]_{q \times q}\left[\begin{array}{cc}
c_{b} & 0 \\
0 & n_{b}
\end{array}\right]_{q \times q} .
$$

Thus, $n_{a}^{*} n_{a}=n_{a}^{*} n_{b}$. Similarly, the second equation implies $n_{a} n_{a}^{*}=n_{b} n_{a}^{*}$. So, $n_{a} \leq^{*} n_{b}$ and therefore $a \leq^{\sharp, *} b$.

(iii) $\Rightarrow\left(\right.$ i) Suppose ${ }^{\circ}\left(b^{k}\right) \subseteq{ }^{\circ}\left(a b^{k}\right)$ and $b^{k} a=a^{k+1}$. Since $a \leq^{\circledast} b$, we have $c_{a} \leq^{\sharp} c_{b}$ and $a \leq^{*} b$. So, $a \leq^{D} b$ and thus by Proposition, there exists a decomposition of the identity $1=e_{1}+e_{2}+e_{3}$ such that

$$
a=\left[\begin{array}{ccc}
c_{1} & 0 & 0 \\
0 & c_{3} & c_{4} \\
0 & c_{5} & c_{6}
\end{array}\right]_{e \times e}, \quad b=\left[\begin{array}{ccc}
c_{1} & 0 & 0 \\
0 & c_{2} & 0 \\
0 & 0 & n_{2}
\end{array}\right]_{e \times e}
$$

where $n_{a}=c_{3}+c_{4}+c_{5}+c_{6}$ is the nilpotent part of $a$ and $n_{2}=n_{b}$ is the nilpotent part of $b$. Here we may without loss of generality assume (see the proof of Theorem 1 ((ii) implies (iii)) in [7]) that $e_{1}=c_{a} c_{a}^{\#}$ and $e_{2}=c_{2} c_{2}^{\#}$. Since $a, b \in \mathfrak{C}^{\mathcal{A}}$, we may (see Remark) as in the proof of Theorem 3 conclude that $e_{1}, e_{2}$, and $e_{3}=1-e_{1}-e_{2}$ are self-adjoined idempotents. Let us prove that $c_{3}=c_{4}=c_{5}=0$. Since $n_{b}^{k}=0=n_{a}^{k}$, we obtain

$$
b^{k} a=\left[\begin{array}{ccc}
c_{1}^{k+1} & 0 & 0 \\
0 & c_{2}^{k} c_{3} & c_{2}^{k} c_{4} \\
0 & 0 & 0
\end{array}\right]_{e \times e} \quad \text { and } \quad a^{k+1}=\left[\begin{array}{ccc}
c_{1}^{k+1} & 0 & 0 \\
0 & 0 & 0 \\
0 & 0 & 0
\end{array}\right]_{e \times e} .
$$

The equation $b^{k} a=a^{k+1}$ yields $c_{2}^{k} c_{3}=0=c_{2}^{k} c_{4}$. It follows that $\left(c_{2}^{\#}\right)^{k} c_{2}^{k} c_{3}=0=$ $\left(c_{2}^{\#}\right)^{k} c_{2}^{k} c_{4}$ and thus $e_{2}^{k} c_{3}=0=e_{2}^{k} c_{4}$. Since $c_{3} \in e_{2} \mathcal{A} e_{2}$ and $c_{4} \in e_{2} \mathcal{A} e_{3}$, we may conclude that $c_{3}=c_{4}=0$. From

$$
e_{3} b^{k}=\left[\begin{array}{ccc}
0 & 0 & 0 \\
0 & 0 & 0 \\
0 & 0 & e_{3}
\end{array}\right]_{e \times e}\left[\begin{array}{ccc}
c_{1}^{k} & 0 & 0 \\
0 & c_{2}^{k} & 0 \\
0 & 0 & 0
\end{array}\right]_{e \times e}
$$

we obtain $e_{3} \in^{\circ}\left(b^{k}\right) \subseteq{ }^{\circ}\left(a b^{k}\right)$. So,

$$
0=\left[\begin{array}{ccc}
0 & 0 & 0 \\
0 & 0 & 0 \\
0 & 0 & e_{3}
\end{array}\right]_{e \times e}\left[\begin{array}{ccc}
c_{1}^{k+1} & 0 & 0 \\
0 & 0 & 0 \\
0 & c_{5} c_{2}^{k} & 0
\end{array}\right]_{e \times e}
$$


and hence $0=e_{3} c_{5} c_{2}^{k}$. Note that $c_{5} \in e_{3} \mathcal{A} e_{2}$. Thus, $c_{5} c_{2}^{k}=0$ and therefore $0=c_{5} c_{2}^{k}\left(c_{2}^{\#}\right)^{k}=c_{5} e_{2}=c_{5}$. It follows that

$$
a=\left[\begin{array}{ccc}
c_{1} & 0 & 0 \\
0 & 0 & 0 \\
0 & 0 & c_{6}
\end{array}\right]_{e \times e}
$$

where $c_{6}=n_{a}$ is the nilpotent part of $a$ and $c_{1}=c_{a}$ is the core part of $a$. Finally, let us show that $n_{a} \leq^{*} n_{b}$. Since $a \leq^{*} b$, we have $a^{*} a=a^{*} b$ and $a a^{*}=b a^{*}$, and thus by (8),

$$
\left[\begin{array}{ccc}
c_{a}^{*} & 0 & 0 \\
0 & 0 & 0 \\
0 & 0 & n_{a}^{*}
\end{array}\right]_{e \times e}\left[\begin{array}{ccc}
c_{a} & 0 & 0 \\
0 & 0 & 0 \\
0 & 0 & n_{a}
\end{array}\right]_{e \times e}=\left[\begin{array}{ccc}
c_{a}^{*} & 0 & 0 \\
0 & 0 & 0 \\
0 & 0 & n_{a}^{*}
\end{array}\right]_{e \times e}\left[\begin{array}{ccc}
c_{a} & 0 & 0 \\
0 & c_{2} & 0 \\
0 & 0 & n_{b}
\end{array}\right]_{e \times e} .
$$

It follows that $n_{a}^{*} n_{a}=n_{a}^{*} n_{b}$. Similarly, we obtain $n_{a} n_{a}^{*}=n_{b} n_{b}^{*}$. So, $n_{a} \leq^{*} n_{b}$ and therefore $a \leq \sharp$,* $b$.

(iv) $\Rightarrow$ (i) We will omit the proof since (by using the matrix formulation (8)) the proof may be very similar to the proof that (iii) implies (i).

(v) $\Rightarrow$ (iii) Let $a b^{k}=b^{k} a=a^{k+1}$ and suppose $z \in{ }^{\circ}\left(b^{k}\right)$. Then $0=z b^{k} a=z a b^{k}$ and therefore ${ }^{\circ}\left(b^{k}\right) \subseteq{ }^{\circ}\left(a b^{k}\right)$.

\section{REFERENCES}

[1] M. P. Drazin, Pseudo-inverses in associative rings and semigroups, Amer. Math. Monthly 65 (1958), 506-514. MR 0098762

[2] M. P. Drazin, Natural structures on semigroups with involution, Bull. Amer. Math. Soc. 84 (1978), no. 1, 139-141. MR 0486234

[3] R. E. Hartwig, J. Luh, A note on the group structure on unit regular ring elements, Pacific J. Math. 71 (1977), no. 2, 449-461. MR 0442018

[4] J. J. Koliha, A generalized Drazin inverse, Glasgow Math. J. 38 (1996), no. 3, 367-381. MR 1417366

[5] J. J. Koliha, P. Patrício, Elements of rings with equal spectral idempotents, J. Aust. Math. Soc. 72 (2002), no. 1, 137-152. MR 1868712

[6] J. Marovt, On partial orders in Rickart rings, Linear Multilinear Algebra 63 (2015), no. 9, 1707-1723. MR 3305004.

[7] J. Marovt, Orders in rings based on the core-nilpotent decomposition, to appear in Linear Multilinear Algebra, http://dx.doi.org/10.1080/03081087.2017.1323846

[8] J. Marovt, D. S. Rakić, D. S. Djordjević, Star, left-star, and right-star partial orders in Rickart *-rings, Linear Multilinear Algebra 63 (2015), no. 2, 343-365. MR 3273759

[9] S. K. Mitra, On group inverses and the sharp order, Linear Algebra Appl. 92 (1987), 17-37. MR 0894635

[10] S. K. Mitra, P. Bhimasankaram, S. B. Malik, Matrix partial orders, shorted operators and applications, Word Scientific, 2010. MR 2647903

[11] D. Mosić, D. S. Djordjević, J. J. Koliha, EP elements in rings, Linear Algebra Appl. 431 (2009), no. 5-7, 527-535. MR 2535530

[12] P. Patrício, R. Puystjens, Drazin-Moore-Penrose invertibility in rings, Linear Algebra Appl. 389 (2004), 159-173. MR 2080402. 
[13] D. S. Rakić, Generalization of sharp and core partial order using annihilators, Banach J. Math. Anal. 9 (2015), no. 3, 228-242. MR 3296136.

[14] D. S. Rakić, N. Č. Dinčić, D. S. Djordjević, Group, Moore-Penrose, core and dual core inverse in rings with involution, Linear Algebra Appl. 463 (2014), 115-133. MR 3262392.

[15] V. Rakočević, Continuity of the Drazin inverse, J. Operator Theory 41 (1999), no. 1, 55-68. MR 1675243

\section{Janko Marovt}

Faculty of Economics and Business, University of Maribor, Razlagova 14, SI-2000 Maribor, Slovenia

janko.marovt@um.si

Received: June 30, 2017

Accepted: October 7, 2017 